\title{
$\operatorname{arCOS} D E S I G N$
}

\section{Temas emergentes na literatura sobre políticas de design}

\section{Gabriel Patrocinio (IFHT/UERJ, Brasil)}

gabrielpatrocinio@gmail.com

UERJ - Instituto Multidisciplinas de Formação Humana com Tecnologias Rua São Francisco Xavier, 524, Prédio anexo - Térreo Maracanã, RJ, Brasil, CEP: 20550-900

Simon Bolton (Birmingham City University, Reino Unido)

simon.bolton@bcu.ac.uk 


\title{
Temas emergentes na literatura sobre políticas de design
}

Resumo: As Políticas Nacionais de Design configuram um campo de importância emergente de pesquisa em design. Procurou-se demonstrar isso através de uma revisão da literatura disponível, coletando evidências de temas, autores e instituições emergentes, e fornecendo uma visão geral de algumas tendências atuais no campo, através de representações visuais sintéticas e análise das informações coletadas. O conhecimento assim gerado, representado visualmente e examinado, oferece espaço para futuras pesquisas no campo.

Palavras-chave: Políticas de Design, promoção de Design, Design e desenvolvimento.

\section{Emerging issues on literature about design policies}

\begin{abstract}
National Design Policies is a field of emerging importance for design research. To demonstrate this, a review of available literature has been done, bringing evidence of emerging themes, authors and institutions, and providing an overview of some current trends in the field. This is achieved with the help of summarised visual representations and analyses of the information collected. This knowledge, visually depicted and examined, offers then further space for future research in the field.
\end{abstract}

Keywords: Design policies, Design promotion, Design and development. 


\section{Introdução}

Os Governos são confrontados hoje com demandas de complexidade crescente oriundas de setores tão distintos quanto desenvolvimento urbano, energia, impactos ambientais, suprimento de alimentos, e benefícios sociais, entre outros. Tais problemas necessitam de abordagem com novos conjuntos de ferramentas que permitam desmembrar essa complexidade em objetivos simples e alcançáveis.

Algumas estratégias de design tem sido consideradas como uma abordagem alternativa para resolver esse problema. Consequentemente, um tema de importância crescente tem sido o das Políticas Públicas de Design - ou como o Governo estabelece princípios para utilizar o design para alavancar o desenvolvimento social, econômico, industrial, e regional. Dois aspectos contribuem para o aumento de interesse no design: a taxa exponencial de crescimento apresentada pelas Industrias Criativas nas ultimas décadas (UNCTAD, 2008; velloso, 2008), e a habilidade do design de servir como um elo entre tecnologia, criatividade e usuário, tendo o potencial de uma ferramenta única para ajudar a inovar e promover economias (HESKETT, 2009; LEE et al., 2007; VINODRAI et al., 2007).

Reconhecendo o papel que o Design pode representar neste cenário, a União Europeia publicou um relatório que enfatiza a necessidade de adequar as Políticas Nacionais e Regionais de Design para que essas contribuam para alavancar mercados - especialmente aqueles mais vulneráveis (CUNNINGHAM, 2008). Diversas agências governamentais e organismos internacionais também tem lançado relatórios, documentos de trabalho e outras publicações sobre o mesmo tema (RAT FOR FORMGEBUng et al., 2010; COMMISSION OF THE EUROPEAN COMMUNITIES, 2009; DESIGN COUNCIL, 2008). Entretanto, muito pouco reconhecimento acadêmico tem sido dedicado a este assunto. Algumas teses de doutoramento recentes indicaram a importância critica de se produzir novos conhecimentos amparados em pesquisa, com a geração de teorias e a consequente busca de evidências (CHOI, 2009; RAUliK-MURPhy, 2009). Outros documentos também se desdobram sobre essa busca por instrumentos apropriados de planejamento e avaliação que dêem suporte à decisões gerenciais de governo (BERNATENE et al., 2009; MOULTRIE-LIVESEY, 2009; CUNNINGHAM, 2008).

Dentro deste contexto, este trabalho enfoca a evolução das políticas nacionais de design, mapeando especificamente os principais autores (tanto indivíduos como organizações), as questões políticas emergentes e as tendências dentro do campo. Estes dados irão possibilitar que pesquisadores e formuladores de políticas estabeleçam de forma mais eficaz os parâmetros de referência para o futuro desenvolvimento de políticas. 


\section{Metodologia}

O presente artigo deriva da analise de um conjunto de dados que continha originalmente 970 documentos, incluindo artigos, livros, relatórios e informes oficiais, coletados durante a revisão empírica de literatura para uma pesquisa doutoral sobre o tema de políticas públicas de design. A maior parte destes documentos foi coletada em formato digital, tendo sido publicados por governos e organizações internacionais.

A partir destes documentos, os autores mais significativos foram apontados - seja pela sua frequência em citações ou pela relevância dos documentos. Alguns nomes aparecem como autores e co-autores em diversos documentos - neste caso destacou-se o autor mais frequente (não necessariamente aquele indicado como autor principal do documento), ou seja, aquele cujo nome aparecia maior número de vezes como autor e co-autor na área. Um exemplo de documento considerado 'relevante' apesar de não ser mencionado muito frequentemente, são os anais do Fórum Mundial de Design "Design Policy and Global Network", promovido pelo Conselho Internacional de Sociedades de Design Industrial - ICSID, e pelo Instituto Coreano de Promoção do Design - KIDP (LEE, 2002), por ser o registro da primeira discussão de relevância internacional sobre o tema no século 21, reunindo artigos que apresentam a temática sob o ponto de vista de diversos atores internacionais. Instituições também são listadas como autoras, quando os nomes dos autores ou editores não são discriminados nos documentos. Este é o caso do Conselho de Design do Reino Unido (Design Council), detentor de extensa produção de relatórios e informes oficiais assinados apenas institucionalmente.

Este recorte, partindo da seleção de autores e instituições, resultou em um subconjunto de 231 documentos, dentre os quais foram identificados 22 temas significantes direta ou indiretamente relacionados ao campo das políticas de design. Alguns temas (assim como autores) contribuíram para estabelecer os referenciais para o campo, como alguns documentos sobre Definições de Design, História do Design, Economia, Inovação, bem como autores como Michael Porter, cujos modelos de competitividade das nações são frequentemente adotados ou citados. Organizações internacionais como o Fórum Econômico Mundial, a Organização para Cooperação e Desenvolvimento Econômico - OCDE, e a Comissão para a Comunidade Europeia, publicaram documentos muito significantes e frequentemente citados sobre inovação, competitividade e desenvolvimento que mencionam habitualmente o papel do design e das politicas de design.

Os temas foram ainda analisados contra uma linha do tempo (segundo suas datas originais de publicação), possibilitando a visualização de agrupamentos temáticos e outros comportamentos ao longo das quatro décadas cobertas pelos documentos coletados. 


\section{Visualizando dados}

Observando-se a representação visual de publicações e citações (Figura 1), alguns nomes sobressaem por diferentes razões: Michael Porter e a sua modelagem da competitividade das nações (PORTER, 1990) é frequentemente usada como referência e citada por muitos autores; outros autores como Bonsiepe, Heskett, Papanek e Thackara contribuíram para estabelecer princípios básicos para o campo. Gui Bonsiepe escreveu diversos documentos, alguns comissionados pelas Nações Unidas, nos anos setenta, fundamentando um discurso do design como ferramenta para o desenvolvimento que ele mais tarde aplicou durante sua experiência em diferentes países da América Latina. Victor Papanek, considerado um pioneiro pelos seus escritos sobre sustentabilidade no início dos anos setenta, fez parte de um grupo de trabalho do ICSID para discutir design para o desenvolvimento. Margolin (2007) afirma que as idéias de Papanek, focadas nas habilidades nativas ou locais, eram opostas às de Bonsiepe, que adotava a ciência e tecnologia como vetores do desenvolvimento. Sir George Cox e seu "Review of Creativity in Business" produzido sob encomenda do primeiro-ministro Gordon Brown em 2005 - é sem dúvida alguma o mais citado na área das políticas de design, e tem sido usado como referencia para diversos outros documentos mais recentes. A Finlândia também é pródiga em autores sobre o tema: Korvenmaa, Valtonen, Hytonen, Nieminen, Saarela. A partir do enquadramento da sua própria proposta de uma política nacional de design, em 2005, muitas pesquisas foram realizadas concentrando-se na Universidade de Artes e Design de Helsinki (atualmente Aalto University), e o seu Centro para Inovação em Design - DESIGNiUm. Alguns dos autores listados (Tether, Swann, Cunningham, Cawood, Raulik-Murphy, Moultrie e outros) desenvolveram pesquisas a partir de demandas de instituições como o Design Council, Nnesta (Fundação Nacional para a Ciência, Tecnologia e Artes - a agência de inovação do Reino Unido), Projeto SEE (Sharing Experience Europe - Policy, Innovation \& Design - uma rede de instituições europeias relacionadas à políticas e promoção de design, baseada na instituição Design Wales, em Cardiff, País de Gales), além da Comissão da Comunidade Europeia. Finalmente, algumas poucas teses acadêmicas foram escritas nos últimos anos em universidades no Reino Unido: H. Alpay Er defendeu sua tese "The Emergence and Development Patterns of Industrial Design in Newly Industrialised Countries with particular reference to Turkey" na Universidade Metropolitana de Manchester em 1994; Youngok Choi apresentou "A Comparative Study of National Design Policy in the UK and South Korea" na Universidade de Lancaster em 2009; e Gisele Raulik-Murphy, autora frequente de artigos na área e gerente do Projeto SEE, defendeu em 
2010 a tese "A Comparative Analysis of Strategies for Design Promotion in Different National Contexts" na Universidade de Wales. Curiosamente, todos não-europeus: Alpay Er é turco, Choi coreana e Raulik-Murphy, brasileira. Isto pode ajudar a entender o foco frequentemente direcionado ao design para o desenvolvimento.

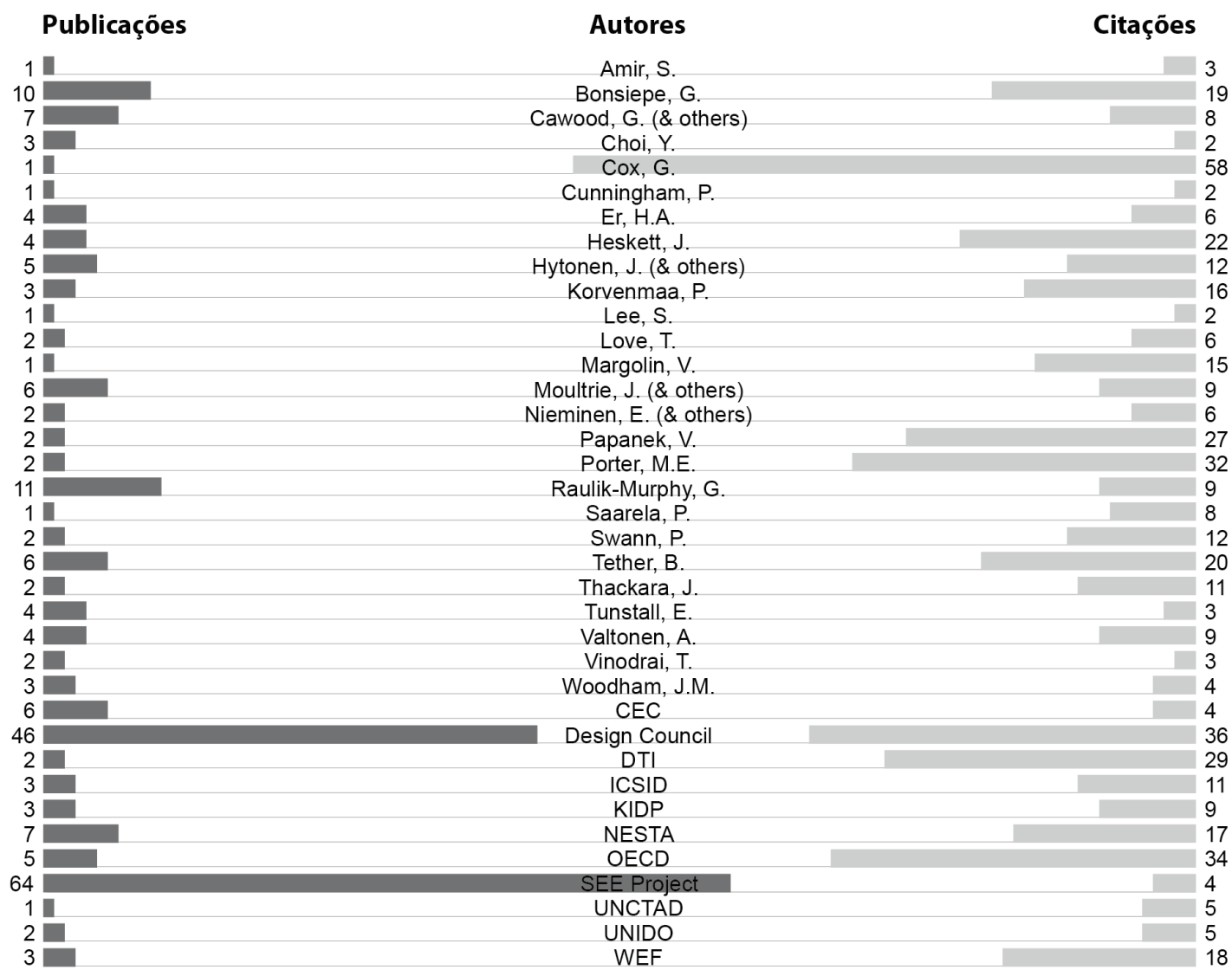

Figura 1. Políticas de design - autores/publicações/citações (fonte: do autor)

Algumas conclusões podem ser extraídas da observação da parte inferior do gráfico de autores/publicações/citações (Figura 1): a primeira e bastante óbvia é a disponibilidade de um grande número de publicações do Design Council. Ele ainda estabelece as bases para discussão na área, seja pelo fato de ter setenta anos de existência (foi criado em 1944) ou efetivamente pelo grande número de publicações que produz. É importante observar que várias publicações do Design Council foram listadas pelo nome dos seus autores, sempre que disponíveis - e portanto as listadas com autoria da instituição foram apenas aquelas aonde o autor não estava identificado. O Projeto SEE é uma outra instituição prolífica, com um bom número de estudos de caso e 'livretos de políticas' (policy booklets) disponíveis no seu website, além da sua revista, 
da qual são editados cerca de quatro números por ano (embora sem periodicidade fixa). O Projeto SEE certamente estendeu o centro de discussão e de produção do conhecimento sobre políticas de design na direção de Wales - ainda que a busca tenha retornado um número baixo de citações. O Departamento de Comércio e Indústria do Reino Unido (Department of Trade and Industry - DTI), é frequentemente citado por outros autores, especialmente pelo documento "Economics Paper No.15 - Creativity, Design and Business Performance". O mesmo ocorre com três documentos da OCDE: "Open Innovation in Global Networks" (2008), "Science, Technology and Innovation Indicators in a Changing World" (2007), e "National Innovation Systems" (1997), frequentemente citados, especialmente quando se podiciona o design dentro de um quadro maior de sistemas de inovação. O Fórum Econômico Mundial (WEF), além de publicar anualmente o seu Global Competitiveness Report, também inseriu diretamente o tema do design a partir do "Global Agenda Council Reports 2010". A NESTA situa o design nos seus relatórios sobre inovação e indústrias criativas, oferecendo suporte para alguns pesquisadores nestas áreas.

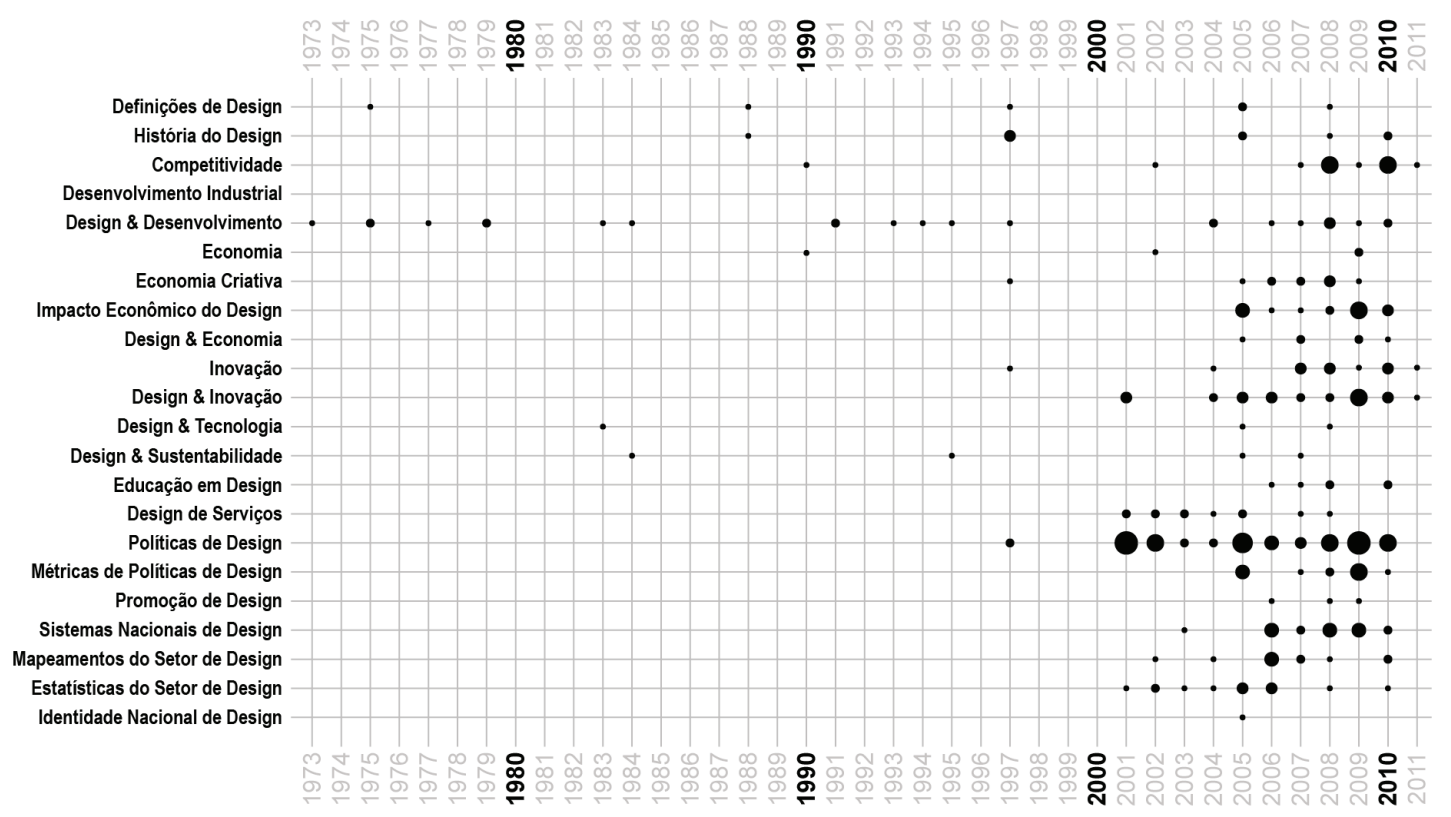

Figura 2. Políticas de design - Cronologia de temas (fonte: do autor)

A partir da representação visual da ocorrência de temas organizados de forma cronológica (Figura 2), primeiro torna-se evidente o quanto a discussão sobre a área se adensou na década de 2000, e especialmente na segunda metade desta. O campo incorporou gradualmente o discurso das Indústrias Criativas / Economia Criativa a partir da segunda metade da década de 2000, 
assim como a importância do Design como vetor da Inovação, e ainda demonstrou o interesse - ou antes a necessidade premente - de métricas, inicialmente através de Mapeamentos e Estatísticas Setoriais e posteriormente temas como o Impacto Econômico do Design e Avaliação de Políticas de Design. Um discurso bastante consistente sobre Design e Desenvolvimento permeou as quatro décadas dos documentos analisados - sendo Bonsiepe o mais constante dos autores nesta área.

O gráfico de Temas e autores (Figura 3) torna mais claros os interesses e as produções individuais, complementando as informações anteriores. Autores como Hytonen, Nieminen, Raulik-Murphy, Saarela, e Tunstall, entre outros, desenvolveram um mapeamento extensivo do setor de design e dos sistemas nacionais de design não apenas nos seus países de origem (Finlândia, Brasil, Estados Unidos), mas também sobre diversos outros, como ferramenta de comparação e aferição. Temas como Educação em Design e Design de Serviços, apesar da sua grande relevância, tem sido pouco explorados - exceto pelo Design Council. Apesar da sua inequívoca importância, o tema Design e Sustentabilidade também tem sido pouco explorado no âmbito das políticas de design.

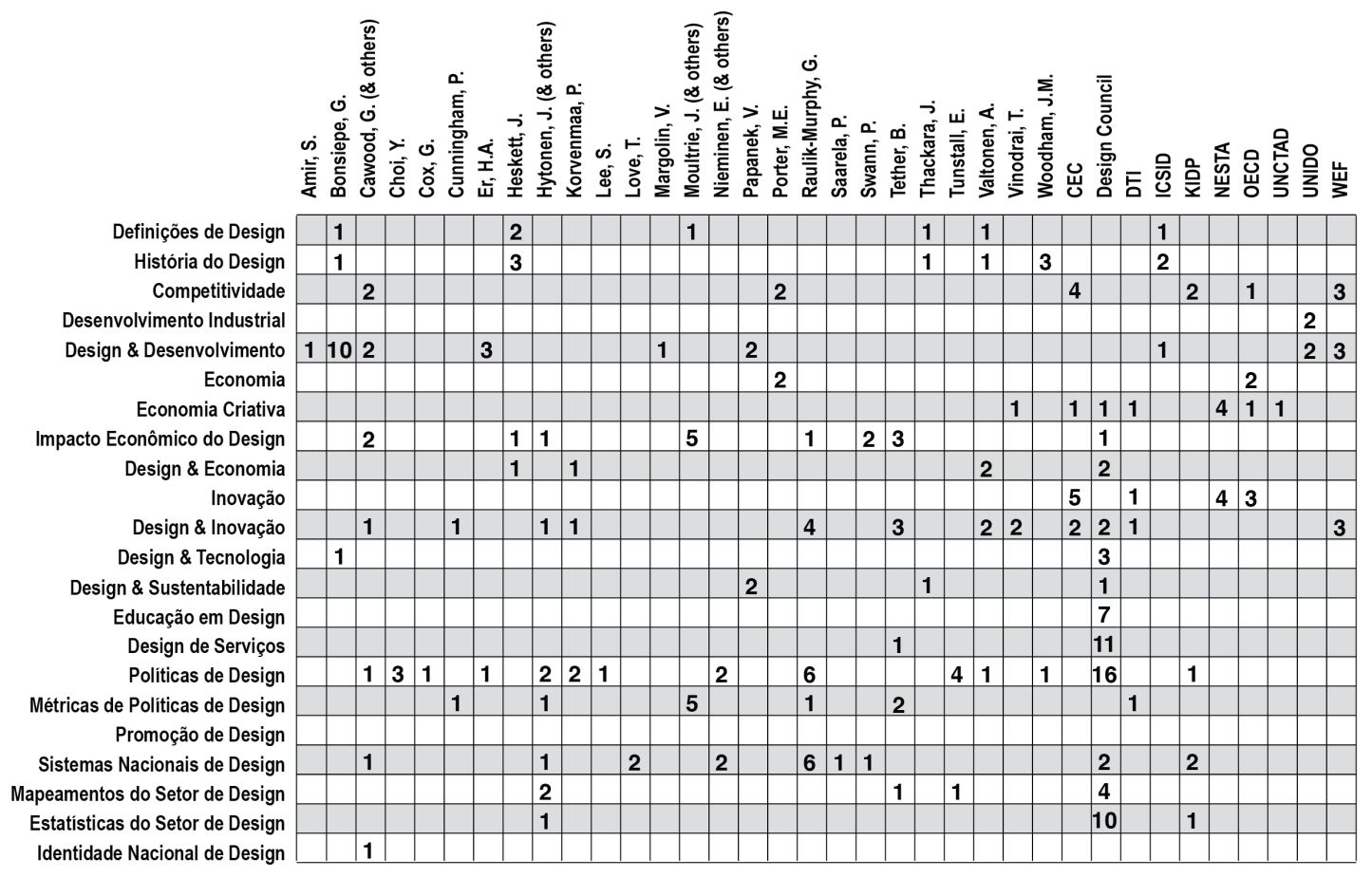

Figura 3. Políticas de design - Temas e autores (fonte: do autor) 


\section{Desdobramentos e condições atuais}

Desde a realização dos estudos iniciais indicados neste artigo (em 2011), a União Europeia tem afirmado seu papel de protagonismo na área de políticas públicas de design, reconhecendo, valorizando e incentivando o papel do design como motor de processos de inovação. Diversos novos documentos foram publicados, dentre os quais destacam-se especialmente quatro: na União Europeia os documentos "Design for Growth \& Prosperity" (тhomson; Koskinen, 2012) e "Design for Public Good" (seE Platform, 2013); no Reino Unido, "Restarting Britain 2 Design and Public Services” (DESIGN COMMISSION, 2013);

e no Brasil, o "Diagnóstico do Design Brasileiro" (свD, 2014). Todos documentos que têm muito a acrescentar ao campo das políticas de design, seja no levantamento de dados e na proposição de métricas (СвD, 2014), ou na proposição mais ampla de políticas nacionais de design (THOMson; KOsKINEN, 2012), e mesmo na proposição de design como ferramenta de construção de políticas e serviços públicos (DEsign commission, 2013). Este último, curiosamente, tem origem numa ação interna do Parlamento Britânico, através do Grupo Parlamentar Associado de Design e Inovação (APDIG, na sigla em inglês), que reúne parlamentares e convida designers para discutir o papel do design nas políticas de governo. Todo este trabalho está também presente e extensamente discutido em outra tese de doutorado recente sobre o assunto (PATROCinio, 2013), que estende a discussão buscando enfocar exemplos do passado - alguns dos quais praticamente desconhecidos - e indicações de possíveis ferramentas para construir e avaliar a performance de políticas públicas de design.

\section{Conclusões e oportunidades para estudos complementares}

Partindo de um conjunto original de dados composto por 970 documentos e chegando a um grupo selecionado de 231 documentos, nos quais 37 autores discorrem sobre 22 temas, esta pesquisa oferece evidências consistentes da importância atribuída atualmente ao tema das Políticas Nacionais de Design. O tema se apresenta em diversos contextos, com autores se originando de diferentes países e experiências. O fato de que o design está sendo considerado por governos e organismos internacionais como uma importante ferramenta de promoção de inovação e desenvolvimento econômico e social fica claramente corroborado. 
As informações aqui presentes pretendem abrir espaço para pesquisas e estudos complementares na área, tais como a importância do design como instrumento de crescimento nas economias em desenvolvimento, ou a busca de métricas eficazes para avaliar a eficiência do design e os resultados das políticas de design. A atualização do estudo, dando a este continuidade com a inclusão de novos documentos publicados entre 2011 e 2014 já se poderia caracterizar como um avanço significativo, servindo ainda mais ao propósito de embasar o desenvolvimento deste novíssimo campo de pesquisa do design.

O entendimento destes fatos oferece uma clara oportunidade para promover uma mudança positiva através de pesquisa em design.

\section{Referências}

BERNATENE, M.; CANALE, G.; CALO, J.; JUSTIANOVICH, S. Nadie puede controlar lo que no mide. In: Instituto Nacional de Tecnologia Industrial (Ed.), Diseño en la Argentina, estudio de impacto economico 2008, p. 61-65, Buenos Aires: INTI, 2009.

CBD CENTRO BRASIL DESIGN. Diagnóstico do Design Brasileiro. Brasilia: ApexBrasil e MDIC, 2014.

CHOI, Y. A Comparative Study of National Design Policy in the UK and South Korea. Lancaster: Lancaster University, 2009. (tese de doutorado não-publicada)

COMMISSION OF THE EUROPEAN COMMUNITIES. Design as a driver of user-centred innovation. Bruxelas: European Commission, 2009.

CUNNINGHAM, P. National and regional policies for design, creativity and user-driven innovation. Manchester: Institute of Innovation Research, Manchester University; Pro Inno Europe, 2008.

DESIGN COMMISSION. Restarting Britain 2 - Design and Public Services. Londres: Policy Connect, 2013.

DESIGN COUNCIL. The Impact of Design on Business. Londres: Design Council, 2008.

HESKETT, J. Creating Economic Value by Design. International Journal of Design, v. 3, n. 1, 2009, p. 71-84.

LEE, S. Design Policy and Global Network: World Design Forum Proceedings. Seul: KIDP; ICSID, 2002. 
LEE, S.; LEE, J.; KIM, B. New design paradigm in the knowledge and creative society. Hong Kong: The Hong Kong Polytechnic University, 2007.

MARGOLIN, V. Design for Development: Towards a History. In: Design Studies, v. 28 n. 2, 2007, p. 111-115.

MOULTRIE, J.; LIVESEY, F. International Design Scoreboard: Initial indicators of international design capabilities. Cambridge: University of Cambridge, Institute for Manufacturing, 2009.

PATROCINIO, G. The Impact of European Design Policies and Their Implications on the Development of a Framework to Support Future Brazilian Design Policies. Cranfield: Cranfield University, School of Applied Sciences, 2013. (tese de doutorado não-publicada)

PORTER, M.E. The Competitive Advantage of Nations. Londres: Macmillan, 1990.

RAT FUR FORMGEBUNG; MARKENVERBAND e.V.; SCHOLZ \& FRIENDS. Die Schönheit des Mehrwertes - The Beauty of Added Value. Berlim: Scholz \& Friends, 2010.

RAULIK-MURPHY, G. A Comparative Analysis of Strategies for Design Promotion in Different National Contexts. Cardiff: The University of Wales, 2009. (tese de doutorado não-publicada).

SEE PLATFORM. Design for Public Good. Londres: Design Council, 2013.

THOMSON, M. \& KOSKINEN, T. Design for Growth and Prosperity. Helsinki: DG Enterprise and Industry of the European Commission, 2012.

UNCTAD. Creative Economy Report 2008 - United Nations Conference on Trade and Development. United Nations, 2008.

VELLOSO, J. P. dos Reis. O Brasil e a economia criativa: um novo mundo nos trópicos. p. 599. Rio de Janeiro: Jose Olympio Editora, 2008.

VINODRAI, T.; GERTLER, M. S.; LAMBERT, R. Capturing Design: Lessons from the United Kingdom and Canada. In: Organisation for Economic Cooperation and Development (ed.), Science, technology and innovation indicators in a changing world, p. 65-85. Paris: OECD Organisation for Economic Cooperation and Development, 2007. 


\section{Como citar}

PATROCINIO, Gabriel; BOLTON, Simon. Temas emergentes na literatura sobre políticas de design. Arcos Design. Rio de Janeiro: PPD ESDI - UERJ. Volume 9 Número 1 Junho 2016. pp. 15-26. Disponível em: [http://www.e-publicacoes.uerj.br/index. php/arcosdesign]

DOI

\section{(c) (1) (3)(2)}

A Revista Arcos Design está licenciada sob uma licença Creative Commons Atribuição - Não Comercial - Compartilha Igual 3.0 Não Adaptada. 